\title{
Autonomous Pick and Place Operations in Industrial Production
}

\author{
Andreas Dömel ${ }^{1}$, Simon Kriegel ${ }^{1}$, Manuel Brucker $^{1}$ and Michael Suppa ${ }^{1}$ \\ ${ }^{1}$ Institute of Robotics and Mechatronics, German Aerospace Center (DLR), 82234 Wessling, Germany \\ (E-mail: andreas.doemel@dlr.de, simon.kriegel@dlr.de, manuel.brucker@dlr.de, michael.suppa@ roboception.de)
}

\begin{abstract}
Pick and place applications are very common in industrial environments. Autonomous execution of this task is desirable, as it is simple and repetitive for a human. To this end, we employ a mobile robot containing a light weight robot (LWR) arm, a pan-tilt unit (PTU) and a multitude of sensors (see Fig. 1).

In production environments, small parts such as screws and nuts are usually transported in a small load carrier (SLC). By manipulating SLCs autonomously, the robot is enabled to assist human workers with various tasks. SLCs used in the manufacturing process of the LWR iiwa, are stored in gravity shelves to guarantee sufficient supply of parts at any time. Restocking these shelves with filled SLCs from the warehouse and returning the empty SLCs is currently carried out by workers. The application of the suggested mobile robot in a real production environment carrying out various pick and place operations has been shown in [1]. In this video, we demonstrate the fundamental abilities of the mobile manipulator needed to carry out an exemplary task fully autonomously. Based on a 2D map, which has to be recorded beforehand, the robot is able to navigate [2] from the warehouse to the production site and vice versa. Due to the fact that the shelves are movable, exact positions cannot be taught. Therefore, the robot has to find the exact relative position to the shelf with its sensor equipment. Using object recognition, environment modeling and path planning the robot is enabled to fully autonomously place filled SLCs into the shelve and retrieve empty ones. At the warehouse, the robot is unloaded and loaded manually by a worker.
\end{abstract}

The Kuka omniRob is a platform with omnidirectional wheels and a LWR4+ mounted on top. As can be seen in [3], most autonomous industrial robot manipulators have not focused on perception. However, the perception of the objects to be manipulated and the environment are essential for application in real environments with uncertainties. Therefore, we integrated multiple sensors on the mobile platform, on a sensor pole with a Schunk PTU and on the robot end effector which enable the system to perceive its environment. On the PTU, a redundant configuration consisting of a ToF camera and a stereo camera system was chosen to overcome sensor specific problems. To enable the system to work without any infrastructure, four I7 processor boards and a FPGA for stereo processing were integrated in the robot. Thus all computations can be performed on board.

As the robot interacts with partially unknown environments such as shelves or the robot's working surface, these need to be explored to allow for collision free path planning. Therefore, the PTU is moved and a proba-

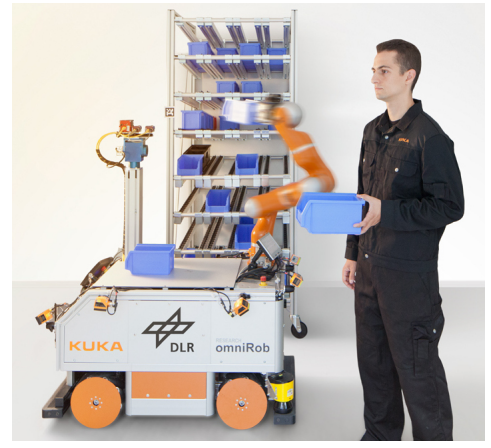

Fig. 1 The DLR omniRob manipulating load carriers.

bilistic voxel space is updated with each depth measurement applying Bayes' Rule as in [4]. Due to the expected scarcity of texture information in industrial scenarios, the object recognition module of the framework is based on depth images. Specifically, a geometric matching approach [4], featuring a fast GPU based verification step, is used. To cope with the high dimensional configuration space of the robot, a sampling based path planner is used for generating autonomous motions. Using existing object models and the sensed environment models, the path planner guarantees collision free motions in partially unknown environments. During manipulation of SLCs, constraints regarding the orientation of the boxes have to be met. Our approach is based on a BiRRT-Connect algorithm using sample rejection for configurations violating the pose constraints.

\section{References}

[1] S. Bogh, C. Schou, T. Ruehr, Y. Kogan, A. Doemel, M. Brucker, C. Eberst, R. Tornese, C. Sprunk, G. D. Tipaldi, and T. Hennessy, "Integration and assessment of multiple mobile manipulators in a real-world industrial production facility," in ISR/Robotik, 2014, pp. 1-8.

[2] J. Röwekämper, C. Sprunk, G. D. Tipaldi, C. Stachniss, P. Pfaff, and W. Burgard, "On the position accuracy of mobile robot localization based on particle filters combined with scan matching," in IEEE/RSJ IROS, 2012, pp. 3158-3164.

[3] M. Hvilshøj, S. Bøgh, O. Skov Nielsen, and O. Madsen, "Autonomous industrial mobile manipulation (AIMM): past, present and future," Industrial Robot, vol. 39, no. 2, pp. 120-135, 2012.

[4] S. Kriegel, M. Brucker, Z.-C. Marton, T. Bodenmüller, and M. Suppa, "Combining Object Modeling and Recognition for Active Scene Exploration," in IEEE/RSJ IROS, 2013, pp. 2384-2391. 